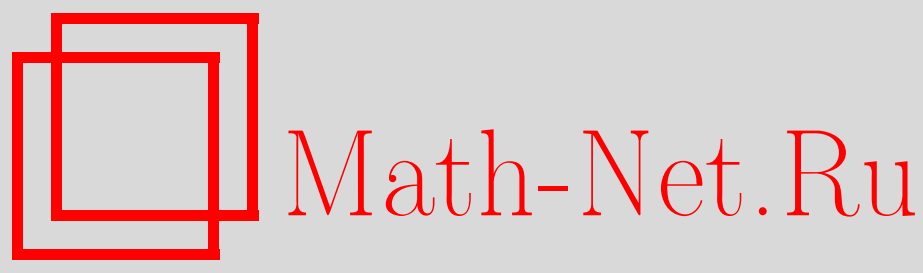

А. Квадри, Канонический поток в пространстве калибровочных параметров, ТМФ, 2015, том 182, номер 1, 91102

DOI: https://doi.org/10.4213/tmf8730

Использование Общероссийского математического портала Math-Net.Ru подразумевает, что вы прочитали и согласны с пользовательским соглашением http://www.mathnet.ru/rus/agreement

Параметры загрузки:

IP : 54.157 .27 .8

26 апреля 2023 г., 04:03:51

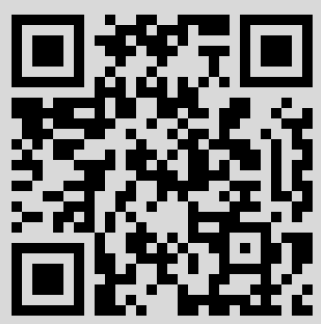




\title{
КАНОНИЧЕСКИЙ ПОТОК В ПРОСТРАНСТВЕ КАЛИБРОВОЧНЫХ ПАРАМЕТРОВ
}

\begin{abstract}
Показано, что зависимость от калибровки для одночастично-неприводимых амплитуд в $S U(N)$-теории Янга-Миллса порождена потоком, каноническим относительно расширенного тождества Славнова-Тейлора, которое индуцировано преобразованием калибровочного параметра $\alpha$ при преобразованиях БРСТсимметрии. В линейных ковариантных калибровках аналитическое разложение одночастично-неприводимых амплитуд по $\alpha$ задается в терминах коэффициентов, вычисляемых в калибровке Ландау, и производных по $\alpha$ от производящего функционала потока. Рассматривается приложение результатов к калибровочному потоку пропагатора глюонов.
\end{abstract}

Ключевые слова: теория Янга-Миллса, БРСТ-симметрия, перенормировка, нелинейная сигма-модель.

DOI: $10.4213 / \operatorname{tmf} 8730$

\section{1. ВВЕДЕНИЕ}

Хотя физические величины и должны быть калибровочно-инвариантными, нередко случается, что одна калибровка оказывается более пригодной, чем другие, с точки зрения практических вычислений при исследовании некоторых свойств калибровочных теорий. В квантовой хромодинамике (КХД) можно найти несколько примеров этого явления. Достаточно отметить некоторые из них - например, эффективное действие в модели конденсата цветного стекла и соответствующие уравнения эволюции легче всего получить в калибровке светового конуса для глюонов со средними скоростями [1]-[3], так что калибровочная инвариантность при этом изначально не очевидна. Тем не менее недавно было доказано [4], что калибровочная инвариантность уравнений эволюции на самом деле сохраняется вследствие выполнения подходящих тождеств Славнова-Тейлора (СТ), возникающих из БРСТ-симметрии в КХД, при наличии классического фонового поля быстрых глюонов, так что фактически для мод глюонов со средними скоростями можно выбирать произвольное калибровочное условие.

*Physical Department, University of Milan, Milan, Italy

${ }^{\dagger}$ INFN - National Institute of Nuclear Physics, Sezione di Milano, Milan, Italy. E-mail: andrea.quadri@mi.infn.it 
Возможно, более удивительным примером оказывается существование массивных решений при подходящих обрезаниях уравнений Швингера-Дайсона в КХД [5]-[7], которое было установлено в калибровке Ландау, что подтвердило результаты вычислений на решетке, выполненные в той же калибровке Ландау, и в случае $S U(2)$ [8], и в случае $S U(3)[9]$. Кроме того, изучение функции Куго-Оджимы также обычно производится в калибровке Ландау [10].

Таким образом, при исследовании инфракрасных свойств КХД представляется особенно важным попытаться найти метод, насколько возможно наиболее общий, который позволяет облегчить сравнения или вычисления, выполняемые в различных калибровках.

С формальной стороны, уже давно известно, что зависимость амплитуд от калибровки можно исследовать алгебраически с помощью (обобщенных) тождеств Нильсена [11]-[13]. Эти тождества можно вывести с помощью продолжения действия БРСТ-дифференциала $s$ на калибровочные параметры. Их БРСТ-вариация задается классическими антикоммутирующими переменными, попарно образующими так называемый БРСТ-дублет (т. е. пару переменных $u, v$ такую, что $s u=v, s v=0$ ). Этот технический прием находится в близкой аналогии с алгебраическим описанием калибровочных теорий в присутствии классического поля связности фонового поля [14]-[17], где снова классическое фоновое поле $\hat{A}_{\mu}$ спаривается под действием БРСТ-дифференциала $s$ с классическим антикоммутирующим полем источника $\Omega_{\mu}$.

Отметим, что данная математическая структура на самом деле возникает весьма естественным образом, если наложить на лежащую в основе калибровочную теорию оба условия: БРСТ- и анти-БРСТ-симметрии [18]. Это требование позволяет получить локальное уравнение антидуха, справедливое в любой лоренц-ковариантной калибровке, путем продолжения локального уравнения антидуха, изначально полученного в калибровке Ландау [19]. Получающееся при этом расширенное тождество СТ, как оказывается, полностью определяет зависимость вершинного функционала $Г$ от фонового поля $\hat{A}_{\mu}$ через каноническое преобразование относительно скобки Баталина-Вилковыского (БВ) в теории, индуцированной производящим функционалом $\Psi_{\mu} \equiv \delta \Gamma / \delta \Omega_{\mu}$ [20], [21]. Кроме того, было показано [22], что решение расширенного тождества СТ можно записать в терминах некоторого ряда Ли, в то время как наивное экспоненцирование может и не сработать в силу зависимости функционала $\Psi_{\mu}$ от фонового поля.

В настоящей работе мы распространяем эти результаты на канонический поток, индуцированный расширенным тождеством СТ, в пространстве калибровочных параметров. Показано, что такой поток можно получить на основе только лишь тождества СТ, поэтому можно абстрагироваться от уравнений, обеспечивающих устойчивость фиксации калибровки при частных выборах калибровочного условия типа уравнений Наканиши-Лаудрупа и уравнений для духов [23] в лоренц-ковариантных калибровках. Далее мы получаем в явном виде ряд Ли, который задает разложение эффективного действия по степеням калибровочного параметра $\alpha$ (в предположении аналитичности по $\alpha$ ). Коэффициенты этого ряда определяются амплитудами, вычисленными в теории при $\alpha=0$ (т. е. в терминах калибровочных амплитуд Ландау в примере лоренц-ковариантных калибровок) с учетом некоторых вкладов, индуцированных зависимостью от параметра $\alpha$ производящего функционала канонического 
потока. Затем мы обсуждаем некоторые особенности калибровочного потока в калибровке Ландау и в лоренц-ковариантной калибровке. Полученные соотношения верны в рамках теории возмущений. Было бы соблазнительно предположить, что эти соотношения могут также оказаться справедливыми и в непертурбативном режиме. Эта задача, однако, выходит далеко за рамки настоящей работы, так как она требует более глубокого обсуждения тех аналитических свойств глюонного пропагатора в пространстве калибровочных параметров вблизи точки Ландау $\alpha=0$, которые могут нарушаться при выходе за рамки теории возмущений.

Работа имеет следующую структуру. В разделе 2 введены обозначения и получено расширенное тождество СТ для $S U(N)$-теории Янга-Миллса. В разделе 3 построен канонический поток, задающий зависимость амплитуд от калибровки, и обсуждается роль зависимости от калибровки для производящего функционала. В разделе 4 проанализирована связь между лоренц-ковариантными калибровками и калибровкой Ландау. Наконец, в разделе 5 обсуждается применение нашего формализма к глюонному пропагатору. Выводы содержатся в заключительном разделе 6 .

\section{2. КЛАССИЧЕСКОЕ ДЕЙСТВИЕ}

Рассмотрим чистую $S U(N)$-теорию Янга-Миллса с классическим действием

$$
S=-\frac{1}{4 g^{2}} \int d^{4} x G_{a \mu \nu}^{2}
$$

и с напряженностью поля, задаваемой формулой

$$
G_{a \mu \nu}=\partial_{\mu} A_{a \nu}-\partial_{\nu} A_{a \mu}+f_{a b c} A_{b \mu} A_{c \nu},
$$

где $f_{a b c}$ суть структурные константы алгебры $S U(N)$. Включение в рассмотрение полей материи не приводит к дополнительным усложнениям в изложенной ниже конструкции.

Обычная процедура квантования, основанная на БРСТ-симметрии, требует введения в древесный вершинный функционал функции $\mathcal{F}_{a}$, фиксирующей калибровку, с помощью спаривания с мультиплетом полей Наканиши-Лаутрупа $b_{a}$ [24]:

$$
S_{\text {g.f. }}=-\int d^{4} x b_{a} \mathcal{F}_{a}
$$

(знак минус введен для удобства обозначений). Для наших целей можно не указывать точный вид функции $\mathcal{F}_{a}$, фиксирующей калибровку. Единственным условием для нее будет то, что она должна позволять обращение двухточечной функции на древесном уровне в секторе $A_{a \mu}-b_{b}$, что позволяет ввести на древесном уровне пропагаторы калибровочных полей и полей мультиплета Наканиши-Лаутрупа. Функция $\mathcal{F}_{a}$ может также зависеть от некоторых параметров. Например, можно выбрать эту функцию в виде

$$
\mathcal{F}_{a}=(1-\lambda) \partial^{\mu} A_{a \mu}+\lambda \partial^{i} A_{i},
$$

интерполирующем между лоренц-ковариантной калибровкой $(\lambda=0)$ и кулоновской калибровкой $(\lambda=1)$. Другой пример дает регуляризация Славнова-Фролова калибровки светового конуса [25]:

$$
\mathcal{F}_{a}=A_{-}+\lambda \partial_{-} A_{-},
$$


где $A_{-}=A_{0}-A_{3}$ и $\partial_{-}=\partial_{0}-\partial_{3}$. Функции Грина вычисляются при $\lambda \neq 0$, после чего следует совершить предельный переход $\lambda \rightarrow 0$.

Калибровочная инвариантность теряется после того, как процедура фиксации калибровки продолжается на полную БРСТ-симметрию с помощью прибавления к классическому действию как члена, фиксирующего калибровку, так и членов, зависящих от полей духов:

$$
S_{\text {g.f. }+ \text { gh }}=s \int d^{4} x \bar{c}_{a}\left(\frac{\alpha}{2} b_{a}-\mathcal{F}_{a}\right)=\int d^{4} x\left(\frac{\alpha}{2} b_{a}^{2}-b_{a} \mathcal{F}_{a}+\bar{c}_{a} s \mathcal{F}_{a}\right),
$$

где нильпотентный БРСТ-дифференциал $s$ действует следующим образом. При действии на калибровочное поле этот дифференциал эквивалентен калибровочному преобразованию с заменой калибровочных параметров на поля духов $c_{a}$ :

$$
s A_{a \mu} \equiv D_{\mu} c_{a}=\partial_{\mu} c_{a}+f_{a b c} A_{b \mu} c_{c}
$$

где через $D_{\mu} c_{a}$ обозначена ковариантная производная поля духов. Закон преобразования для поля духов, в свою очередь, диктуется условием нильпотентности дифференциала $s$, т. е.

$$
s c_{a}=-\frac{1}{2} f_{a b c} c_{b} c_{c} .
$$

Поле антидуха $\bar{c}_{a}$ и поля мультиплета Наканиши-Лаутрупа $b_{a}$ образуют дублет относительно БРСТ-преобразований [26], [27]:

$$
s \bar{c}_{a}=b_{a}, \quad s b_{a}=0 .
$$

Параметр $\alpha$ редуцируется к известному калибровочному параметру для лоренцковариантных калибровок, когда $\mathcal{F}_{a}=\partial A_{a}$.

2.1. Тождество Славнова-Тейлора. Поскольку БРСТ-вариации калибровочного поля и полей духов в уравнениях (2) и (3) нелинейны по квантовым полям, их перенормировка требует введения внешних источников, известных как антиполя [28]. Они связаны с БРСТ-вариациями соответствующих полей:

$$
S_{\text {a.f. }}=\int d^{4} x\left(A_{a \mu}^{*} s A_{a \mu}-c_{a}^{*} s c_{a}\right) .
$$

Знак минус перед $c_{a}^{*} s c_{a}$ введен для совместности выражений с соглашениями для скобки БВ из работы [28].

Тогда вершинный функционал на древесном уровне

$$
\Gamma^{(0)}=S+S_{\text {g.f. }+ \text { gh }}+S_{\text {a.f. }}
$$

удовлетворяет тождеству СТ [29], [30]

$$
\mathcal{S}\left(\Gamma^{(0)}\right) \equiv \int d^{4} x\left(\frac{\delta \Gamma^{(0)}}{\delta A_{a \mu}^{*}} \frac{\delta \Gamma^{(0)}}{\delta A_{a \mu}}-\frac{\delta \Gamma^{(0)}}{\delta c_{a}^{*}} \frac{\delta \Gamma^{(0)}}{\delta c_{a}}+b_{a} \frac{\delta \Gamma^{(0)}}{\delta \bar{c}_{a}}\right)=0 .
$$


Заметим, что, поскольку БРСТ-преобразование поля антидуха $\bar{c}_{a}$ линейно, введение антиполя для $\bar{c}_{a}$ не обязательно.

Тождество СТ (5) выполняется вне зависимости от конкретного выбора функции $\mathcal{F}_{a}$, фиксирующей калибровку. Для некоторых частных выборов последней (например, для линейных ковариантных калибровок или для калибровки Ландау) возникают дополнительные тождества типа уравнения для поля $b$ и уравнения для духа [23]. Однако эти дополнительные тождества в дальнейшем применяться не будут.

2.2. Вариация БРСТ-калибровочных параметров. Давно известно [12], что можно продолжить действие БРСТ-симметрии на пространство калибровочных параметров и таким образом получить расширенные тождества СТ, которые приводят к так называемым тождествам Нильсена [11], [12]. Положим

$$
s \lambda_{i}=\theta_{i}, \quad s \theta_{i}=0, \quad s \alpha=\theta, \quad s \theta=0 .
$$

При действии расширенной БРСТ-симметрии в выражении (1) для действия $S_{\text {g.f. }+ \text { gh }}$ возникает дополнительный член,

$$
\begin{aligned}
S_{\text {g.f. }+ \text { gh }} & =s \int d^{4} x \bar{c}_{a}\left(\frac{\alpha}{2} b_{a}-\mathcal{F}_{a}\right)= \\
& =\int d^{4} x\left(\frac{\alpha}{2} b_{a}^{2}-b_{a} \mathcal{F}_{a}+\bar{c}_{a} s \mathcal{F}_{a}-\bar{c}_{a} \frac{\theta}{2} b_{a}\right)+\int d^{4} x \bar{c}_{a}\left(\frac{\partial \mathcal{F}_{a}}{\partial \lambda_{i}} \theta_{i}+\frac{\partial \mathcal{F}_{a}}{\partial \alpha} \theta\right),
\end{aligned}
$$

и при этом классическое действие на древесном уровне удовлетворяет расширенному тождеству СТ:

$$
\widetilde{\mathcal{S}}\left(\Gamma^{(0)}\right)=\sum_{i} \theta_{i} \frac{\partial \Gamma^{(0)}}{\partial \lambda_{i}}+\theta \frac{\partial \Gamma^{(0)}}{\partial \alpha}+\mathcal{S}\left(\Gamma^{(0)}\right)=0 .
$$

Для неаномальных теорий это равенство выполнено для полного вершинного функционала Г:

$$
\widetilde{\mathcal{S}}(\Gamma)=\sum_{i} \theta_{i} \frac{\partial \Gamma}{\partial \lambda_{i}}+\theta \frac{\partial \Gamma}{\partial \alpha}+\mathcal{S}(\Gamma)=0 .
$$

Вычисляя производную по $\theta$ и полагая затем переменные $\theta, \theta_{i}$ равными нулю, получим тождество Нильсена

$$
\begin{aligned}
\left.\frac{\partial \Gamma}{\partial \alpha}\right|_{\theta=\theta_{i}=0}= & -\int d^{4} x\left(\frac{\delta^{2} \Gamma}{\partial \theta \delta A_{a \mu}^{*}} \frac{\delta \Gamma}{\delta A_{a \mu}}-\frac{\delta \Gamma}{\delta A_{a \mu}^{*}} \frac{\delta^{2} \Gamma}{\partial \theta \delta A_{a \mu}}-\right. \\
& \left.-\frac{\delta^{2} \Gamma}{\partial \theta \delta c_{a}^{*}} \frac{\delta \Gamma}{\delta c_{a}}-\frac{\delta \Gamma}{\delta c_{a}^{*}} \frac{\delta^{2} \Gamma}{\partial \theta \delta c_{a}}+b_{a} \frac{\delta^{2} \Gamma}{\partial \theta \delta \bar{c}_{a}}\right)\left.\right|_{\theta=\theta_{i}=0}
\end{aligned}
$$

Похожее равенство имеет место и для производной функции $Г$ по $\lambda_{i}$, если вычислить производную расширенного тождества СТ (6) по $\theta_{i}$. 


\section{3. КАНОНИЧЕСКИЙ ПОТОК ДЛЯ КАЛИБРОВОЧНЫХ ПАРАМЕТРОВ}

Имеется близкая формальная аналогия между уравнением (6) и расширенным тождеством СТ, которое описывает зависимость от конфигураций фонового поля $\hat{A}_{\mu}$ [14]-[17]. Эта аналогия связана с тем, что и калибровочные параметры, и конфигурации фонового поля образуют БРСТ-дублеты (т.е. пары переменных $u, v$ таких, что $s u=v, s v=0)$ со своими БРСТ-партнерами.

Предполагая аналитическую зависимость от конфигураций фонового поля, можно получить решение расширенных тождеств СТ в виде подходящего ряда Ли [22], который позволяет выразить все коэффициенты разложения по степеням поля $\hat{A}_{\mu}$ с помощью функций Грина, вычисленных при нулевом фоновом поле. То обстоятельство, что простое экспоненцирование не работает и приходится применять ряд Ли, связано с зависимостью производящего функционала, задающего зависимость от фонового поля, от собственно фонового поля.

С помощью той же техники можно получить ряд Ли для разложения вершинного функционала по степеням $\alpha$ (или $\lambda_{i}$ ) в терминах амплитуд, вычисленных при $\alpha=0$ (или $\lambda_{i}=0$ ). При этом снова надо предполагать аналитическую зависимость от параметра $\alpha$ (или $\lambda_{i}$ ). Для этого удобно переписать расширенное тождество СТ в рамках формализма БВ [28]. Для этого введем антиполе $\bar{c}_{a}^{*}$ для антидуха $\bar{c}_{a}$, а также антиполе $b_{a}^{*}$ для поля Наканиши-Лаутрупа $b_{a}$. Поле $\bar{c}_{a}^{*}$ взаимодействует с полем $b_{a}$ в классическом действии, в то время как поле $b_{a}^{*}$ не входит в $\Gamma^{(0)}$ (поскольку $\left.s b_{a}=0\right)$.

Определим теперь скобку БВ следующим образом (используются только левые производные):

$$
\{X, Y\}=\int d^{4} x \sum_{\phi}\left[(-1)^{\epsilon_{\phi}\left(\epsilon_{X}+1\right)} \frac{\delta X}{\delta \phi} \frac{\delta Y}{\delta \phi^{*}}-(-1)^{\epsilon_{\phi^{*}}\left(\epsilon_{X}+1\right)} \frac{\delta X}{\delta \phi^{*}} \frac{\delta Y}{\delta \phi}\right] .
$$

Здесь суммирование производится по полям $\phi=\left(A_{a \mu}, c_{a}, \bar{c}_{a}, b_{a}\right)$ и по соответствующим антиполям $\phi^{*}=\left(A_{a \mu}^{*}, c_{a}^{*}, \bar{c}_{a}^{*}, b_{a}^{*}\right)$. Через $\epsilon_{\phi}, \epsilon_{\phi^{*}}$ обозначены статистики поля $\phi$ и антиполя $\phi^{*}$, а через $\epsilon_{X}$ - статистика функционала $X$.

Теперь расширенное тождество СТ (6) можно записать в виде

$$
\widetilde{\mathcal{S}}(\Gamma)=\sum_{i} \theta_{i} \frac{\partial \Gamma}{\partial \lambda_{i}}+\theta \frac{\partial \Gamma}{\partial \alpha}+\frac{1}{2}\{\Gamma, \Gamma\}=0 .
$$

Взяв производную по $\theta$ (при этом аргумент ведет себя так же, как и при дифференцировании по $\left.\theta_{i}\right)$, получим

$$
\left.\frac{\partial \Gamma}{\partial \alpha}\right|_{\theta=\theta_{i}=0}=-\left.\left\{\frac{\partial \Gamma}{\partial \theta}, \Gamma\right\}\right|_{\theta=\theta_{i}=0} .
$$

Это соотношение показывает, что производная вершинного функционала по $\alpha$ получается с помощью канонического преобразования (относительно скобки БВ), индуцированного производящим функционалом $\Psi \equiv \partial \Gamma / \partial \theta$. Поскольку этот производящий функционал в общем случае зависит от $\alpha$, уравнение (7) не удается решить 
простым экспоненцированием и приходится прибегать к разложениям в ряды Ли. Для этого введем оператор

$$
\Delta_{\Psi}=\{\cdot, \Psi\}+\frac{\partial}{\partial \alpha} .
$$

Тогда вершинный функционал Г будет задаваться следующим рядом Ли [22]:

$$
\Gamma=\left.\sum_{n \geqslant 0} \frac{1}{n !} \alpha^{n}\left[\Delta_{\Psi}^{n} \Gamma_{0}\right]\right|_{\alpha=0},
$$

где $\Gamma_{0}-$ вершинный функционал при $\alpha=0$. Заметим, что после этого необходимо произвести предельный переход $\alpha \rightarrow 0$ (хотя оператор $\Delta_{\Psi}$ и применяется к функционалу $\Gamma_{0}$, который не зависит от $\alpha$ ), поскольку все еще может оставаться остаточная зависимость от $\alpha$ (и такая зависимость в самом деле остается в общем случае), которая происходит из дифференцирования производящего функционала $\Psi$ по $\alpha$.

Отметим также, что уравнение (8) выполняется вне зависимости от вида функции $\mathcal{F}_{a}$, фиксирующей калибровку (и, в частности, не зависит от существования уравнения для полей $b$ или уравнения для духов, гарантирующих устойчивость условия фиксации калибровки в определенных классах калибровочных условий [23]).

\section{4. ЛОРЕНЦ-КОВАРИАНТНЫЕ КАЛИБРОВКИ}

Проиллюстрируем вышеописанный формализм на простом примере лоренц-ковариантной калибровки, т. е. выберем

$$
\mathcal{F}_{a}=\partial A_{a}
$$

Тогда имеем

$$
S_{\text {g.f. }+ \text { gh }}=\int d^{4} x\left(\frac{\alpha}{2} b_{a}^{2}-b_{a} \partial A_{a}+\bar{c}_{a} \partial^{\mu}\left(D_{\mu} c\right)_{a}+\frac{\theta}{2} \bar{c}_{a} b_{a}\right) .
$$

Пропагаторы имеют вид

$$
\begin{aligned}
\Delta_{A_{\mu}^{a} A_{\nu}^{b}} & =-i \delta^{a b}\left(\frac{1}{p^{2}} T^{\mu \nu}+\frac{\alpha}{p^{2}} L^{\mu \nu}\right), & \Delta_{b_{a} A_{b \mu}} & =-\delta^{a b} \frac{p^{\mu}}{p^{2}}, \\
\Delta_{b_{a} b_{b}} & =0, & \Delta_{c_{a} \bar{c}_{b}} & =\delta_{a b} \frac{i}{p^{2}} .
\end{aligned}
$$

Здесь $T^{\mu \nu}=g^{\mu \nu}-p^{\mu} p^{\nu} / p^{2}-$ поперечный проектор, а $L^{\mu \nu}=p^{\mu} p^{\nu} / p^{2}-$ продольный проектор.

В случае лоренц-ковариантных калибровок справедливы следующие уравнения для полей $b$ и духов:

$$
\frac{\delta \Gamma}{\delta b_{a}}=\alpha b_{a}-\partial A_{a}, \quad \frac{\delta \Gamma}{\delta \bar{c}_{a}}=\partial^{\mu} \frac{\delta \Gamma}{\delta A_{a \mu}^{*}}-\frac{\theta}{2} b_{a} .
$$

Вследствие первого из этих уравнений зависимость от $b$ ограничена на древесном уровне. Из второго уравнения, в свою очередь, следует, что в старших порядках $n \geqslant 1$ функционал $\Gamma$ может зависеть от $\bar{c}_{a}$ только через комбинацию

$$
\tilde{A}_{a \mu}^{*}=A_{a \mu}^{*}-\partial_{\mu} \bar{c}_{a} .
$$

4 Теоретическая и математическая физика, т. 182, № 1, 2015 г. 
Переопределив антиполе $A_{a \mu}^{*}$ в соответствии с приведенным выше равенством и введя редуцированный функционал

$$
\widetilde{\Gamma}=\Gamma-\int d^{4} x \frac{\alpha}{2} b_{a}^{2}+\int d^{4} x b_{a} \partial A_{a}
$$

можно ограничить скобку БВ на переменные $\left(A_{a \mu}, \tilde{A}_{a \mu}^{*}\right)$ и $\left(c_{a}, c_{a}^{*}\right)$; при этом уравнение потока принимает вид

$$
\left.\frac{\partial \widetilde{\Gamma}}{\partial \alpha}\right|_{\theta=\theta_{i}=0}=-\int d^{4} x\left(\frac{\delta \Psi}{\delta A_{a \mu}} \frac{\delta \widetilde{\Gamma}}{\delta \tilde{A}_{a \mu}^{*}}-\frac{\delta \Psi}{\delta \tilde{A}_{a \mu}^{*}} \frac{\delta \widetilde{\Gamma}}{\delta A_{a \mu}}+\frac{\delta \Psi}{\delta c_{a}} \frac{\delta \widetilde{\Gamma}}{\delta c_{a}^{*}}-\frac{\delta \Psi}{\delta c_{a}^{*}} \frac{\delta \widetilde{\Gamma}}{\delta c_{a}}\right) .
$$

Поскольку в дальнейшем используется только функционал $\widetilde{\Gamma}$, вместо него будем просто писать $Г$. Оператор Ли $\Delta_{\Psi}$ имеет вид

$$
\begin{aligned}
\Delta_{\Psi}(X) & =\{X, \Psi\}+\frac{\partial X}{\partial \alpha}= \\
& =\int d^{4} x\left(\frac{\delta X}{\delta A_{a \mu}} \frac{\delta \Psi}{\delta \tilde{A}_{a \mu}^{*}}-\frac{\delta X}{\delta \tilde{A}_{a \mu}^{*}} \frac{\delta \Psi}{\delta A_{a \mu}}+\frac{\delta X}{\delta c_{a}} \frac{\delta \Psi}{\delta c_{a}^{*}}-\frac{\delta X}{\delta c_{a}^{*}} \frac{\delta \Psi}{\delta c_{a}}\right)+\frac{\partial X}{\partial \alpha} .
\end{aligned}
$$

Вследствие уравнения (9) на древесном уровне $\Psi$ редуцируется к виду

$$
\Psi=\int d^{4} x \frac{1}{2} \bar{c}_{a} b_{a}+O(\hbar) .
$$

В данном случае $\Gamma_{0}$ в уравнении (8) представляет собой вершинный функционал теории Янга-Миллса в калибровке Ландау. С помощью уравнения (8) при этом можно выразить коэффициенты разложений по $\alpha$ одночастично-неприводимых амплитуд в лоренц-ковариантной калибровке в терминах одночастично-неприводимых амплитуд в калибровке Ландау с добавлением вклада, зависящего от $\alpha$ и задающегося производящим функционалом $\Psi$.

Коэффициент $\Gamma_{1}$ получается в соответствии с уравнением (8) с помощью однократного применения оператора $\Delta_{\Psi}$ к $\Gamma_{0}$. Так как $\Gamma_{0}$ не зависит от $\alpha$, имеем

$$
\Gamma_{1}=\left.\int d^{4} x\left(\frac{\delta \Gamma_{0}}{\delta A_{a \mu}} \frac{\delta \Psi}{\delta \tilde{A}_{a \mu}^{*}}-\frac{\delta \Gamma_{0}}{\delta \tilde{A}_{a \mu}^{*}} \frac{\delta \Psi}{\delta A_{a \mu}}+\frac{\delta \Gamma_{0}}{\delta c_{a}} \frac{\delta \Psi}{\delta c_{a}^{*}}-\frac{\delta \Gamma_{0}}{\delta c_{a}^{*}} \frac{\delta \Psi}{\delta c_{a}}\right)\right|_{\alpha=0} .
$$

Это выражение задает линейное приближение функционала Г в разложении по степеням калибровочного параметра $\alpha$ в терминах амплитуд, вычисленных в калибровке Ландау.

Перейдем к вычислению коэффициента $\Gamma_{2}$, который определяется как

$$
\Gamma_{2}=\left.\frac{\partial^{2} \Gamma}{\partial \alpha^{2}}\right|_{\alpha=0}
$$

В соответствии с уравнением (8) можно найти $\Gamma_{2}$, если дважды применить $\Delta_{\Psi}$ к $\Gamma_{0}$, а затем положить $\alpha=0$. При этом получаются два члена. Первый снова содержит 
только амплитуды в калибровке Ландау, и его можно согласованно записать в виде $\left.\left\{\Psi,\left\{\Psi, \Gamma_{0}\right\}\right\}\right|_{\alpha=0}$. Этот член связан с простым экспоненцированием. Однако также возникает вклад от производной функционала $\Psi$ по $\alpha$, так что полный вид $\Gamma_{2}$ таков:

$$
\begin{aligned}
\Gamma_{2}= & \left.\left\{\Psi,\left\{\Psi, \Gamma_{0}\right\}\right\}\right|_{\alpha=0}+ \\
& +\left.\int d^{4} x\left(\frac{\delta \Gamma_{0}}{\delta A_{a \mu}} \frac{\delta^{2} \Psi}{\partial \alpha \delta \tilde{A}_{a \mu}^{*}}-\frac{\delta \Gamma_{0}}{\delta \tilde{A}_{a \mu}^{*}} \frac{\delta^{2} \Psi}{\partial \alpha \delta A_{a \mu}}+\frac{\delta \Gamma_{0}}{\delta c_{a}} \frac{\delta^{2} \Psi}{\partial \alpha \delta c_{a}^{*}}-\frac{\delta \Gamma_{0}}{\delta c_{a}^{*}} \frac{\delta^{2} \Psi}{\partial \alpha \delta c_{a}}\right)\right|_{\alpha=0} .
\end{aligned}
$$

\section{5. ЗАВИСИМОСТЬ ГЛЮОННОГО ПРОПАГАТОРА ОТ КАЛИБРОВКИ}

В качестве примера рассмотрим, как можно в пертурбативном режиме вывести решение калибровочного эволюционного уравнения для поперечной составляющей глюонного пропагатора. Для этого введем поперечные и продольные формфакторы

$$
\Delta_{A_{\mu}^{a} A_{\nu}^{b}}=-i \delta^{a b}\left(\Delta_{T}\left(p^{2}\right) T^{\mu \nu}+\Delta_{L}\left(p^{2}\right) L^{\mu \nu}\right) .
$$

Искомая величина - это $\Delta_{T}\left(p^{2}\right)$. Вычисляя вторую производную уравнения (10) по $A_{b_{1} \nu_{1}}, A_{b_{2} \nu_{2}}$ и полагая после этого все поля и внешние источники равными нулю, получим

$$
\frac{\partial \Gamma_{A_{b_{1} \nu_{1}} A_{b_{2} \nu_{2}}}}{\partial \alpha}=-\int d^{4} x\left(\Gamma_{\theta \tilde{A}_{a \mu}^{*} A_{b_{1} \nu_{1}}} \Gamma_{A_{b_{2} \nu_{2}} A_{a \mu}}+\Gamma_{\theta \tilde{A}_{a \mu}^{*} A_{b_{2} \nu_{2}}} \Gamma_{A_{b_{1} \nu_{1}} A_{a \mu}}\right) .
$$

В этом равенстве использовались короткие обозначения, в которых нижние индексы обозначают функциональные производные по соответствующему аргументу, и подразумевается, что после произведения всех дифференцирований все поля $\Phi$ и внешние источники $\Phi^{*}, \theta, \theta_{i}$ полагаются равными нулю. Например,

$$
\left.\Gamma_{A_{b_{1} \nu_{1}} A_{b_{2} \nu_{2}}} \equiv \frac{\delta^{2} \Gamma}{\delta A_{b_{1} \nu_{1}} \delta A_{b_{2} \nu_{2}}}\right|_{\Phi=\Phi^{*}=\theta=\theta_{i}=0} .
$$

Введем поперечные и продольные формфакторы для наших одночастично-неприводимых функций, а именно (в фурье-пространстве)

$$
\begin{aligned}
\Gamma_{A_{b_{1} \nu_{1}} A_{b_{2} \nu_{2}}} & =\delta_{b_{1} b_{2}}\left(G^{T} T_{\mu \nu}+G^{L} L_{\mu \nu}\right), \\
\Gamma_{\theta \tilde{A}_{a \mu}^{*} A_{b \nu}} & =\delta_{a b}\left(R^{T} T_{\mu \nu}+R^{L} L_{\mu \nu}\right) .
\end{aligned}
$$

Тогда, действуя поперечным проектором на уравнение (11), получим

$$
\frac{\partial G^{T}}{\partial \alpha}=-2 R^{T} G^{T}
$$

Обозначим через $G_{0}^{T}$ формфактор в калибровке Ландау. При этом, интегрируя уравнение (12), получим

$$
G^{T}=\exp \left(-\int_{0}^{\alpha} d \alpha^{\prime} 2 R^{T}\right) G_{0}^{T}
$$


и потому для поперечной составляющей глюонного пропагатора имеет место формула

$$
\Delta^{T}=\exp \left(\int_{0}^{\alpha} d \alpha^{\prime} 2 R^{T}\right) \Delta_{0}^{T} .
$$

С другой стороны, в соответствии с выражением (13) отношение

$$
r=\exp \left(-\int_{0}^{\alpha} d \alpha^{\prime} 2 R^{T}\right) \frac{\Delta^{T}}{\Delta_{0}^{T}}
$$

должно быть равно единице (а потому быть калибровочно-инвариантным).

Хотя наши результаты и справедливы в рамках теории возмущений, их продолжение вне пределов этой теории представляет собой тонкий вопрос, далеко выходящий за рамки рассмотрения в настоящей работе.

Некоторые вычисления в калибровке Ландау, основанные на уравнениях Швингера-Дайсона, на самом деле позволяют отождествить масштабированное решение при $\Delta_{T}(0)=0$ [31] с отщепленным решением при $\Delta_{T}(0)>0$ (см. работы [5], [6] и ссылки в них). Поэтому представляется важным исследовать, что происходит с этими классами решений при калибровочных вариациях, т. е. сравнить эволюцию с имеющимися сегодня результатами вычислений на решетке при $\alpha \neq 0$ [32].

Если бы можно было взять инфракрасный предел в обеих частях уравнения (13), то из равенства $\Delta_{0}^{T}(0)=0$ следовало бы, что $\Delta_{T}(0)$ также обращается в ноль. Таким образом, если бы решение уравнения Швингера-Дайсона имело масштабированный вид в калибровке Ландау, оно имело бы тот же тип в лоренц-ковариантной калибровке. Более того, для массивных решений знак $\Delta_{T}(0)$ не зависел бы от калибровки вследствие уравнения (13). Однако справедливость уравнения (13) вне рамок теории возмущений вызывает сомнения. В частности, наличие инфракрасных расходимостей в явных непертурбативных вычислениях формфактора $R^{T}$ может привести к нарушению предположения об аналитической зависимости амплитуд вблизи калибровочной точки Ландау $\alpha=0$. В этом случае решение (8) в виде ряда Ли нельзя использовать при реконструкции вершинного функционала в калибровке с $\alpha \neq 0$.

\section{6. ЗАКЛЮЧИТЕЛЬНЫЕ ЗАМЕЧАНИЯ}

Существование канонического потока в пространстве калибровочных параметров и связанное с ним решение в терминах ряда Ли позволяют сравнивать результаты в различных калибровках в рамках алгебраического подхода, справедливого также и вне рамок теории возмущений (по крайней мере до тех пор, пока остается справедливым тождество СТ).

Зависимость производящего функционала канонического потока от калибровочного параметра не позволяет получить полное решение с помощью простого экспоненцирования. Такое решение можно выразить в терминах подходящего ряда Ли в тесной аналогии с решением расширенного тождества СТ в присутствии фоновой калибровочной связности.

Знание такого ряда Ли упрощает процедуру сравнения вычислений, производящихся в различных калибровках. В простейшем примере двухточечной глюонной функции можно получить в замкнутом виде формулу, интерполирующую между 
калибровкой Ландау и лоренц-ковариантной калибровкой, в предположении об аналитичности зависимости от калибровочного параметра в окрестности точки $\alpha=0$.

Благодарности. Автор глубоко признателен Д. Биноси за критическое прочтение рукописи.

\section{Список литературы}

[1] E. Iancu, A. Leonidov, L. D. McLerran, Nucl. Phys. A, 692:3-4 (2001), 583-645, arXiv: hep-ph/0011241.

[2] E. Ferreiro, E. Iancu, A. Leonidov, L. McLerran, Nucl. Phys. A, 703:12 (2002), 489-538, arXiv: hep-ph/0109115.

[3] Y. Hatta, E. Iancu, L. McLerran, A. Staśto, D. N. Triantafyllopoulos, Nucl. Phys. A, 764 (2006), 423-459, arXiv: hep-ph/0504182.

[4] D. Binosi, A. Quadri, D. N. Triantafyllopoulos, High-energy QCD evolution from BRST symmetry, arXiv: 1402.4022 .

[5] A. C. Aguilar, D. Binosi, J. Papavassiliou, Phys. Rev. D, 78:2 (2008), 025010, 6 pp., arXiv: 0802.1870.

[6] D. Binosi, J. Papavassiliou, Phys. Rep., 479:1-6 (2009), 1-152, arXiv: 0909.2536.

[7] C. S. Fischer, A. Maas, J. M. Pawlowski, Ann. Phys., 324:11 (2009), 2408-2437, arXiv: 0810.1987.

[8] A. Cucchieri, T. Mendes, PoS (LATTICE 2007), 2007, 297, 7 pp., arXiv: 0710.0412.

[9] I. L. Bogolubsky, E. M. Ilgenfritz, M. Müller-Preussker, A. Sternbeck, Phys. Lett. B, 676:1-3 (2009), 69-73, arXiv: 0901.0736.

[10] A. C. Aguilar, D. Binosi, J. Papavassiliou, JHEP, 11 (2009), 066, 29 pp., arXiv: 0907.0153.

[11] N. K. Nielsen, Nucl. Phys. B, 101:1 (1975), 173-188.

[12] O. Piguet, K. Sibold, Nucl. Phys. B, 253 (1985), 517-540.

[13] P. Gambino, P. A. Grassi, Phys. Rev. D, 62:7 (2000), 076002, 17 pp., arXiv: hep-ph/9907254.

[14] P. A. Grassi, Nucl. Phys. B, 462:2 (1996), 524-550, arXiv: hep-th/9505101.

[15] P. A. Grassi, Nucl. Phys. B, 560:1-3 (1999), 499-550, arXiv: hep-th/9908188.

[16] C. Becchi, R. Collina, Nucl. Phys. B, 562:1 (1999), 412-430, arXiv: hep-th/9907092.

[17] R. Ferrari, M. Picariello, A. Quadri, Ann. Phys., 294:2 (2001), 165-181, arXiv: hep-th/0012090.

[18] D. Binosi, A. Quadri, Phys. Rev. D, 88:8 (2013), 085036, 13 pp., arXiv: 1309.1021.

[19] P. A. Grassi, T. Hurth, A. Quadri, Phys. Rev. D, 70:10 (2004), 105014, 12 pp., arXiv: hep-th/0405104.

[20] D. Binosi, A. Quadri, Phys. Rev. D, 84:6 (2011), 065017, 16 pp., arXiv: 1106.3240.

[21] D. Binosi, A. Quadri, Phys. Rev. D, 85:8 (2012), 085020, 12 pp., arXiv: 1201.1807.

[22] D. Binosi, A. Quadri, Phys. Rev. D, 85:12 (2012), 121702,5 pp., arXiv: 1203.6637.

[23] O. Piguet, S. P. Sorella, Algebraic renormalization. Perturbative Renormalization, Symmetries and Anomalies, Lecture Notes in Physics. New Series m: Monographs, 28, Springer, Berlin, 1995.

[24] N. Nakanishi, Progr. Theor. Phys., 35:6 (1966), 1111-1116; B. Lautrup, Mat. Fys. Medd. Kon. Dan. Vid.-Sel. Medd., 35 (1967), 29.

[25] А. А. Славнов, С. А. Фролов, ТМФ, 73:2 (1987), 199-209.

[26] G. Barnich, F. Brandt, M. Henneaux, Phys. Rep., 338:5 (2000), 439-569, arXiv: hep-th/0002245. 
[27] A. Quadri, JHEP, 05 (2002), 051, 16 pp., arXiv: hep-th/0201122.

[28] J. Gomis, J. Paris, S. Samuel, Phys. Rep., 259:1-2 (1995), 1-145, arXiv: hep-th/9412228.

[29] А. А. Славнов, ТМФ, 10:2 (1972), 153-161.

[30] J. C. Taylor, Nucl. Phys. B, 33:2 (1971), 436-444.

[31] R. Alkofer, L. von Smekal, Phys. Rep., 353:5-6 (2001), 281-465, arXiv: hep-ph/0007355.

[32] A. Cucchieri, T. Mendes, G. M. Nakamura, E. M. S. Santos, PoS (FacesQCD), 2010, 026, 10 pp., arXiv: 1102.5233. 